\title{
ALUMNADO UNIVERSITARIO CON DISCAPACIDAD: ELEMENTOS PARA LA REFLEXIÓN PSICOPEDAGÓGICA
}

\section{UNIVERSITY STUDENT WITH DISABILITY: ELEMENTS FOR THE PSYCHOPEDAGOGY REFLECTION}

\author{
Diego Jesús Luque Parra* y Gemma Rodríguez Infante** \\ Universidad de Málaga
}

\section{RESUMEN}

En este artículo se tratan aspectos de la situación educativa universitaria del alumnado con discapacidad. En él se busca situar elementos de intervención psicopedagógica, a través de la actuación llevada a cabo por un Servicio de Apoyo al Alumnado con discapacidad. Con todo se pretende en última instancia, favorecer la reflexión sobre el papel de la Orientación Educativa, el Profesorado y la Comunidad Universitaria en general, en su relación con la discapacidad.

Palabras clave: Universidad. Discapacidad. Orientación Educativa. Servicio de Apoyo.

\section{ABSTRACT}

This article talks about different aspects of disable students at university level. It focuses on the psichopedagogical intervention from the Support Service for Students with Disabilities. The final goal is to promote the reflection on the role of educational guidance, teachers and university staff as a whole in relation with disabilities.

Key words: University, Disability, Educational Guidance, Support Service's.

\section{Introducción}

En todos los sistemas educativos europeos, con independencia de la obligatoriedad y caracteres específicos de formación, incluida la enseñanza universitaria como una posible

\footnotetext{
* Profesor del Dpto. de Psicología Evolutiva y de la Educación, y Profesor Especialista del Servicio de Apoyo al Alumnado con Discapacidad, (SAAD). Universidad de Málaga. Departamento de Psicología Evolutiva y de la Educación, Universidad de Málaga. Campus de Teatinos, (29071) Málaga. Correo-E: dluque@uma.es

** Licenciada en Psicopedagogía. Master en Atención Temprana. Universidad de Málaga. Oficina Técnica del Servicio de Apoyo al Alumnado con Discapacidad. Universidad de Málaga. (29071) Campus de El Ejido. gri@uma.es
} 
etapa más en la carrera del estudiante, ha adquirido suma importancia la atención al alumnado en su diversidad y al que presenta discapacidad en particular. Así, a lo largo de la década de los noventa del pasado siglo, se han producido cambios en todos los países, en su legislación educativa hacia la Integración, con un acercamiento que deja ser exclusivamente médico, para enmarcarse en lo educativo (como ámbito de unificación, coordinación e intervención) hacia las personas con discapacidad (Agencia Europea para el Desarrollo de la Educación Especial, 1998; Hegarty, 1996). Por otro lado, en el ámbito del Espacio Europeo de Educación Superior, tanto en la Declaración de La Sorbona (1998), como en la de Bolonia (1999), se promueve una dimensión de la Educación Superior, particularizando en el desarrollo curricular, cooperación institucional y en programas integrados de estudios, de formación y de investigación. Posteriormente, en el Comunicado de Praga (2001), en una de sus líneas adicionales a las Declaraciones anteriores, se introduce que el aprendizaje es a lo largo de toda la vida, como elemento esencial para alcanzar, entre otros aspectos, la mejora de la cohesión social, la igualdad de oportunidades y la calidad de vida (MECD, 2003).

La Universidad, aunque se regula por sus normas específicas, constituye un tramo superior de la enseñanza (Ley Orgánica 2/2006, de 3 de mayo, de Educación; BOE 106 de 04/05/2006), por lo que es un paso más en la estructura educativa y, en consecuencia, otro tipo de respuesta a las necesidades del Alumnado, aunque corre el riesgo de perder continuidad en diversos aspectos, más incluso que en los sucesivos cambios de nivel, ciclos o etapas educativas anteriores. Así, considerando que estas divisiones no son más que un recurso operativo y/o administrativo, en lugar de acentuar las diferencias entre etapas, deberían pensarse en términos de seguimiento de desarrollo y plena formación de la persona, viéndose a la Universidad, como una continuidad a la Educación Secundaria post-obligatoria (integral y no exclusivamente académica o profesional). Si esto puede ser así para el conjunto de la población estudiantil, es especialmente preocupante para los alumnos con necesidades especiales, en los que deberá evitarse que el símil de escalón-progreso, pueda convertirse en otro de muro-limitación, por lo que, y con un desenvolvimiento en un ámbito educativo inclusivo, superador de integración y solidaridad en la educación, son necesarios los mecanismos de compensación y adaptación, facilitadores del desarrollo personal del alumnado con discapacidad. Se cumplen así los preceptos constitucionales, desde los que la respuesta de la Universidad al alumnado con discapacidad, tiene dos frentes de actuación. De un lado, la atención al desarrollo de sus capacidades e intereses, a través de los elementos de compensación y adaptación curricular; y por otro, la facilitación de los elementos de acceso y dominio de situaciones, con los que alcanzar niveles de igualdad con sus compañeros sin dificultades. Recomendaciones que se recogen en la Resolución del Consejo (5 de mayo de 2003) de la Unión Europea, sobre la igualdad de oportunidades en educación y formación para los alumnos y estudiantes con discapacidad (DOC 134 de 7.6.2003).

Puede decirse que la Universidad ha presentado un cierto déficit en la atención al alumnado con discapacidad, sin que ello haya significado falta de preocupación o interés, sino más bien actuaciones específicas o en áreas de intervención (trabajo social, ayudas, apoyos particulares, etc.), con menor conjunción o integración. En este sentido, podría añadirse que la Universidad ha comenzado de perder ese carácter deficitario en el tratamiento educativo y/o en la formación en la discapacidad (actuaciones psicopedagógicas, legislativas o de experiencias en general), desde que la voluntad de trabajo y realizaciones prácticas, llevados a cabo por un grupo de Universidades españolas, bajo el patrocinio del Real Patronato sobre 
Discapacidad, ha conseguido construir una estructura y dinámica de integración educativa universitaria digna de elogios. Las Universidades de Valencia, Extremadura, Autónoma de Barcelona, UNED, Málaga, etc., son ejemplos pioneros de las experiencias en este terreno. En este sentido, el presente artículo, desde la experiencia y actuaciones del Servicio de Apoyo al Alumnado con Discapacidad de la Universidad de Málaga, propone un acercamiento a este alumnado, con el que hacer algunas reflexiones sobre su intervención psicopedagógica.

\section{Alumnado con discapacidad y universidad}

Desde la perspectiva de la Clasificación Internacional del Funcionamiento, la Discapacidad y la Salud (CIFDS, OMS. 2001), por discapacidad se entiende la circunstancia de aspectos negativos de la interacción del individuo y sus factores contextuales, limitaciones de la actividad y restricciones de la participación. Se distancia de la concepción de la Clasificación Internacional de Deficiencia, Discapacidad y Minusvalía (OMS, 1983), anclada en lo médico o distintividad por la deficiencia, para situarse en una visión en la que el funcionamiento y la discapacidad, forman un proceso de interacción recíproca, en un curso vital y cuyo resultado o unión es superior a la suma de sus partes. En consecuencia, la discapacidad es aceptada como un estado o situación, en el que se tiene menor grado de habilidad o ejecución en el desarrollo de capacidades, debido a una interacción de factores individuales y de contexto (Luque y Rodríguez, 2005; Luque, 2006).

En un ámbito exclusivamente educativo, la discapacidad se valora y trata centrándose en la adecuación a las necesidades y características de la persona, valorándose sus aspectos, a fin de compensar con medidas y recursos, de forma que, la discapacidad, se anula o reduce de la mano de una intervención o provisión de servicios y apoyos. En este ámbito, podría ser indistinto el uso de los términos discapacidad o de personas o alumnado con necesidades especiales (nees), con los que, además de proseguir la línea educativa de etapas anteriores, se centran en lo contextual y en la respuesta educativa y, al asociarse a la discapacidad, hace que ésta se considere, en su mayor parte, una condición sobrevenida por el contexto. Estas necesidades son especiales no por un trastorno o distintividad, sino por la atención a las dificultades y a los recursos que se precisan y, por ello, dejarían de serlo si la docencia, los recursos y el ajuste curricular, tuvieran un carácter ordinario en los centros, lo cual nos remite a factores de contexto (accesibles y/o compensadores) que anularían o reducirían la discapacidad o limitaciones de la persona con su medio.

Es obvio que no todos los alumnos y alumnas con discapacidad (la tengan o no reconocida), tendrán necesidades especiales (cuadro 1), en la medida que la provisión de servicios que necesiten sean adecuados a su situación y circunstancia, pero sí a la inversa, todo el alumnado con necesidades especiales, presentará algún elemento de discapacidad (necesitada de ayuda cuando lo solicite o las limitaciones que se presenten puedan impedir o dificultar su desenvolvimiento). En cualquier caso, las dificultades de las personas y sus necesidades educativas especiales, son concreciones de las necesidades individuales y, como tales, deberán contemplarse en los objetivos educativos y formativos de las áreas y materias de enseñanza. 
CUADRO 1: Necesidades educativas especiales en la universidad. Luque y Rodríguez, 2005.

Supresión de barreras arquitectónicas: rampas de acceso, puertas con dimensiones establecidas, ascensores, servicios adaptados, plazas de aparcamiento, etc.

Mobiliario y/o espacios adaptados.

Ordenadores y recursos informáticos en general, adecuados.

Organización y ubicación de servicios: biblioteca, lugares comunes de ocio, conserjería, etc.

Intérprete de lengua de signos.

Uso del Braille.

Acompañante.

Exámenes adaptados: tipo test, orales, preguntas grabadas, etc.

Pruebas complementarias: actividades diarias, trabajos, etc.

Tiempo extra para ejecución y terminación de tareas.

Comprensión y apoyo de tribunales.

Textos grabados, tanto sonoros como en braille.

Unidades Didácticas individualizadas. Informatización de materiales didácticos. Facilitación de apuntes.

Tutorías personalizadas.

Orientación y Consejo Psicológico.

Ayudas económicas de libros, transporte y materiales de apoyo.

Vehículo de apoyo para desplazamientos

Apoyo de entidades, asociaciones y grupos.

Asesoramiento laboral.

Otros.

Desde la igualdad y la equidad, como valores de ajuste social y en un desarrollo educativo o formativo de la persona, el alumnado con discapacidad se sitúa en una circunstancia particular de necesidades especiales, porque precisan de recursos o modificaciones en el currículum, que compensen su situación académica y normalicen su desarrollo formativo. Propugnando una acción formativa y de intervención psicopedagógica desde un modelo de diseño universal, serían mínimas o innecesarias adaptaciones o individualizaciones (connotaciones de excepcionalidad), desarrollándose una pedagogía sin exclusión, en la que las necesidades no tendrían adjetivos específicos, sólo los propios de los intereses asociados al sustantivo de la persona del alumno, en su contexto y desarrollo. 


\section{Respuesta docente e intervención psicopedagógica}

La respuesta a esas necesidades en la Universidad, se contempla desde el análisis de las capacidades individuales y, en menor grado de las dificultades de aprendizaje que pudieran plantear, todo ello con los matices distintivos a las presentadas en los tramos educativos no universitarios. Además, el acercamiento legislativo a las necesidades educativas especiales (nees) en la Universidad, se ha hecho de una forma general, y recogida en una normativa mínima, en su mayor parte, a efectos de matriculación y acceso, no habiéndose profundizado en un desarrollo de mayor alcance psicopedagógico, académico y formativo, que haga operativa la educación del Alumnado con discapacidad. Así, frente a un desarrollo educativo de los alumnos y alumnas con necesidades especiales, regulado y estructurado en los tramos de Educación Infantil, Primaria y Secundaria, en los estudios universitarios, salvo en lo contemplado, de forma genérica en el R. D. 696/1995, de 28 de abril, de Ordenación de la Educación de los Alumnos con nees puede decirse que se carece de legislación específica ${ }^{2}$. Sin embargo, la Ley Orgánica 4/2007, de 12 de abril, por la que se modifica la ley Orgánica 6/2001, de diciembre, de Universidades destaca en su disposición adicional vigésimo cuarta, de la inclusión de las personas con discapacidad en las Universidades, todo un texto de considerable importancia y reflexión, para la garantía de igualdad de oportunidades en la inclusión de personas con discapacidad en las universidades (anexo). Disposición que supone todo un marco de propuestas y base de consideración en los Estatutos de la propias Universidades.

En consecuencia, se impone la valoración de un desarrollo normativo más aplicado o reconocedor de la discapacidad en la Universidad en general, así como la contemplación de esa circunstancia en sus Estatutos y normativas en particular. De esta forma, se tratará de evitar que, en la atención al alumnado con discapacidad, se consideren como meras declaraciones, ni que se queden en "paquetes" de atención específica, sino propuestas de atención del sistema, sin concepciones de distintividad o excepcionalidad, en un marco de igualdad real.

El Profesorado universitario, con una actuación docente basada en la reflexión y sensibilidad hacia la discapacidad (cuadro 2), sólo debe atender a esas necesidades de su alumnado, aportando los elementos de formación y de trabajo de su asignatura, de forma accesible o con las adaptaciones que correspondan, en función del ajuste individual. Así, las adaptaciones que se hagan del currículum, al menos inicialmente, se dirigirán hacia la accesibilidad y los recursos, quedando las adaptaciones internas al currículum, en una consideración detenida de su significatividad. Esto es, las modificaciones curriculares se centrarán en lo metodológico, actividades, evaluación y contenidos no nucleares, no afectándose los contenidos fundamentales y los objetivos. La cuestión del nivel de las enseñanzas, está en íntima relación con la consecución de la titulación profesional válida y homologada, con lo que, la adecuación al alumnado con discapacidad, ha de salvaguardar la adquisición y desarrollo de las competencias básicas para los distintos ejercicios profesionales, lo cual no es óbice para

\footnotetext{
1. Pueden citarse, como normativa posterior al respecto, orientada en su mayor parte hacia elementos de ordenación académica, el RD. 1640/99, de 22 de Octubre (BOE del 27) y el RD. 69/2000, de 21 de enero (BOE del 22), por los que se regulan la adaptación de pruebas y la reserva del 3\% de plazas disponibles, respectivamente. Asimismo se expresa la terminología de estudiantes con NEEs permanentes asociadas a las condiciones personales de discapacidad que durante su escolarización anterior hayan precisado recursos extraordinarios.
} 
que el alumno realice los estudios de la carrera que ha elegido y para la que superado criterios de selectividad.

\section{CUADRO 2. Principios para una actuación docente en la discapacidad. Elaboración propia.}

Aceptar la Educación como medio de desarrollo personal y social, afianzándose siempre en el núcleo de la persona del alumno.

Generar una enseñanza y un aprendizaje en el desarrollo de capacidades y habilidades.

Asimilar la discapacidad como hecho o estado, resultado de la interacción individuo-contexto.

Apreciar la accesibilidad y la adaptación como mecanismos de desarrollo, compensación y ajuste de la persona y alumno con discapacidad.

Desarrollar una práctica educadora justa, adecuada y en consideración a las características del alumno, dentro de valores de respeto a la diferencia, cooperación y el apoyo mutuo entre las personas.

Los Alumnos con necesidades especiales, en consecuencia, pueden acceder y desarrollar sus estudios en la Universidad, debiendo considerarse las adaptaciones que se estimen pertinentes, ya que institucionalmente se ha de disponer de los aspectos relativos a la ordenación, planificación y organización de recursos y atención, con los que esos alumnos (con necesidades temporales o permanentes) puedan alcanzar los objetivos establecidos con carácter general para todo el alumnado. Puesto que la mayor parte de estos alumnos, ya tiene experiencia o conocimiento de los programas de integración de los Centros no universitarios anteriores, sus evaluaciones psicopedagógicas, habrán puesto de manifiesto unas capacidades y desarrollo cognitivo propias para el aprendizaje y enseñanzas universitarias. En gran parte serán personas con discapacidad sensorial o motórica, siendo menores los que presenten discapacidad psíquica y más reducido aún, los de deficiencia intelectual, lo cual no deja de poner de manifiesto, las relaciones entre desarrollo evolutivo, cognitivo y académico, así como los sucesivos "filtros" en el sistema educativo, previos al ingreso en la Universidad, a la que esos alumnos acceden, habiendo superado las pruebas establecidas con carácter general, para el conjunto del alumnado.

Si nos preguntamos por el papel del Psicopedagogo, la respuesta es la de actuar desde un ámbito de Orientación Educativa, prosiguiendo así la realizada en las etapas anteriores no universitarias. En efecto, la Intervención Psicopedagógica, como elemento operativo deberá centrarse en lo nuclear de la persona del alumno y su progreso, en un marco de accesibilidad y en la compensación de dificultades o limitaciones, esto es, actuar desde un modelo de Intervención Psicopedagógico interactivo y contextual. De esta forma, los principios de normalización (condiciones de igualdad) e integración (respeto, aceptación y apoyo), no son más que los referentes propios de una Universidad inclusiva, que expresa y participa de la comunidad a la que sirve.

Las funciones generales de evaluación (proceso y resultado de obtención de información para toma de decisiones) e intervención (proyecto que busca obtener resultados en los procesos con el alumno y contexto), que el psicopedagogo realiza, se convierten en el ámbito universitario y alumnos con discapacidad, en dos procesos determinados, valoración y asesoramiento (cuadro 3). 
Cuadro 3. Aspectos psicopedagógicos generales. Luque y Rodríguez, 2005.

\begin{tabular}{|c|l|l|}
\cline { 2 - 3 } \multicolumn{1}{c|}{} & \multicolumn{1}{c|}{ Evaluación } & \multicolumn{1}{c|}{ Intervención } \\
\hline \multirow{2}{*}{ Alumnado } & $\begin{array}{l}\text { Identificación y valoración de } \\
\text { necesidades y recursos. } \\
\text { Características individuales, sociales } \\
\text { y de contexto. }\end{array}$ & $\begin{array}{l}\text { Aspectos de desarrollo personal y social. } \\
\text { Compensación de dificultades y } \\
\text { promoción. } \\
\text { Seguimiento de Programas. }\end{array}$ \\
\hline $\begin{array}{c}\text { Profesorado } \\
\text { - Centro }\end{array}$ & $\begin{array}{l}\text { Detección y valoración de recursos y y } \\
\text { programas. }\end{array}$ & $\begin{array}{l}\text { Asesoramiento en discapacidad y en de contextos. } \\
\text { Informes o Dictámenes } \\
\text { desarrollo curricular. } \\
\text { Programas y adaptaciones. }\end{array}$ \\
\hline Familia & Valorapedagógicos. & \\
\hline
\end{tabular}

De acuerdo a lo anterior, la Intervención Psicopedagógica en la discapacidad, trataría la consecución del objetivo general en la Universidad, aplicar el principio de igualdad de oportunidades, compensando las necesidades educativas, formando a la persona y favoreciendo el acceso y desarrollo de los estudios universitarios. En todo caso, la enseñanza universitaria debe conducirse desde patrones de aceptación, comprensión y apoyo al alumnado, en términos de accesibilidad y facilitación de recursos y de adaptación, dentro de un diseño universal, premisas con las que la atención se desenvuelva en un medio justo y reconocedor de su persona y circunstancia.

\section{Aspectos aplicados de intervención psicopedagógica}

En la Universidad de Málaga, el Servicio de Apoyo al Alumnado con Discapacidad (SAAD), supone el núcleo de intervención psicopedagógica, pretendiendo servir tanto a los alumnos y alumnas, como al profesorado en sus actuaciones y relaciones de enseñanza y aprendizaje. Como se ha señalado (Luque, Elósegui y Rodríguez, 2005, p. 7), y desde ese núcleo de actuación, se ha de plantear el objetivo general de "Garantizar la igualdad de oportunidades y la plena integración de los estudiantes universitarios con discapacidad en la vida académica universitaria, además de promover la sensibilización y la concienciación del resto de miembros de la comunidad universitaria". Objetivo general que se hace operativo en los siguientes objetivos específicos:

- Asesorar académicamente a los estudiantes y profesores en aquellas cuestiones relacionadas con la discapacidad y los estudios universitarios.

- Informar sobre recursos educativos, legislativos y económicos con los que cuentan los estudiantes con discapacidad durante su etapa formativa.

- Sensibilizar y concienciar a toda la comunidad universitaria con la finalidad de potenciar la plena integración de la persona con discapacidad, no sólo en el contexto universitario sino en el conjunto de la sociedad. 
- Valorar las necesidades individuales que planteen los alumnos con necesidades educativas especiales.

- Adaptar el currículum académico de los estudiantes con discapacidad atendiendo a sus necesidades específicas.

El alumnado de la Universidad de Málaga que expresa su discapacidad en su matrícula (curso 2005/06), tiene la distribución que sigue.

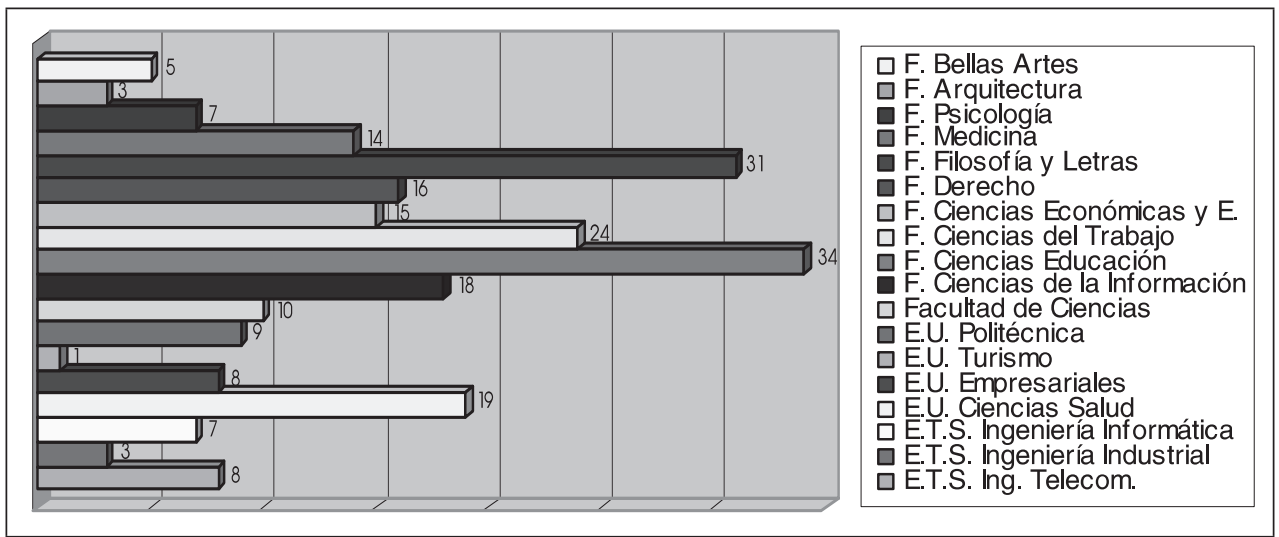

FIGURA 1.

Matrícula de alumnos con discapacidad. Luque y Rodríguez, 2006.

De un total de 232 alumnos, han solicitado ayuda en el Servicio de Apoyo, 71 estudiantes, lo que representa un 30\% aproximadamente, desfase típico en todas las Universidades, españolas y europeas, que podría deberse a que el alumno no quiere que se sepa o le resta importancia a su discapacidad, o como si ésta supusiera alguna dificultad llevadera y no encasillarse como alumno discapacitado, prefiriendo vivir su condición como un alumno más. La respuesta del Servicio ha sido amplia, en función de las necesidades individuales. Así se han llevado a cabo las siguientes actuaciones.

Pueden apreciarse las diferentes actuaciones, en función de las características personales del alumnado. De los 71 alumnos solicitantes, se mantuvieron 36 entrevistas de asesoramiento, de éstos, 34 recibieron un tratamiento más personalizado $\mathrm{y}$, en doce casos, se ha asesorado a los padres sobre los recursos que ofrece la Universidad. Además, el SAAD ha realizado actuaciones de orientación profesional en alumnos de secundaria que han solicitado asesoramiento, ante las inminentes pruebas de selectividad. En otro apartado, cuando se cree conveniente, se asesora al profesorado de determinados alumnos para informarle de sus características y de las adaptaciones curriculares necesarias, asesoramiento que se ha llevado a cabo en un total de 10 alumnos.

Junto a estas medidas, los alumnos con discapacidad de la Universidad de Málaga, cuando así lo precisan, cuentan con diferentes apoyos, como la figura de un alumno colaborador (becario y compañero de clase que ayudaría al alumno con discapacidad, en sus tareas diarias en la Universidad), cuidadores (personal cualificado encargado de asistir o acompañar al alumno), ayudas económicas para el transporte y préstamo de ayudas técnicas. 
Tabla 1. Actuaciones del Servicio de Apoyo al Alumnado con Discapacidad. Elaboración propia.

\begin{tabular}{|l|c|}
\hline \multicolumn{1}{|c|}{ Actuaciones del saad } & $\mathbf{N}^{\mathbf{0}}$ alumnos \\
\hline Orientación vocacional & 3 \\
\hline Entrevistas personales & 71 \\
\hline Asesoramiento alumnos & 36 \\
\hline Asesoramiento padres & 12 \\
\hline Valoracion psicopedagogica & 18 \\
\hline Asesoramiento profesores & 10 \\
\hline Alumno colaborador & 6 \\
\hline Cuidador & 1 \\
\hline Ayudas al transporte & 4 \\
\hline Interprete lengua signos & 2 \\
\hline Ayudas técnicas & 3 \\
\hline
\end{tabular}

Se hace obvio expresar que sin estas medidas, estos alumnos se situarían en una posición de desventaja con respecto al resto de sus compañeros, y muchos no podrían seguir con sus estudios universitarios por falta de recursos, de aquí que, la Intervención Psicopedagógica en la Universidad, deba ser considerada fundamental en el caso de alumnos con necesidades especiales derivadas de su discapacidad, continuando así el proceso educativo de la Etapa Secundaria.

\section{Conclusiones}

La Universidad, como Institución supone una forma de convivencia y cauce para el desarrollo de la persona, en la que, en el caso de los alumnos con discapacidad, la igualdad como valor de ajuste social, ha de concretarse en el principio de no discriminación (igualdad de todas las personas ante las leyes), además de su materialización en la discriminación positiva, como fenómeno de compensación, y en un principio de realidad (igualdad real o material en la vida social), facilitando el esfuerzo de cada persona, no sustituyéndolo, sino haciéndola protagonista de su historia personal y social. A modo de resumen final, podríamos concluir en algunos puntos de reflexión docente sobre la discapacidad, en el ámbito universitario:

- La Universidad es la continuidad al esfuerzo de la Comunidad Educativa y del alumnado con nees, para desarrollar su persona y su formación profesional como cualquier otro ciudadano, al que en cambio, es preciso compensar en sus dificultades y características personales, todo ello, desde una perspectiva de normalidad y no sólo de su discapacidad. Esto será un hecho educativo (normalización e integra- 
ción) en la medida que, la institución que acoja a los alumnos, se provea de recursos y atención, ajustando el proceso de currículum-alumnado.

- Desde la perspectiva de una sociedad democrática, con planteamientos educativos universales y de justicia distributiva, no pueden permitirse situaciones en las que algún alumno o alumna, tengan disminuida su accesibilidad y desarrollo a los estudios superiores. Desde esos mismos postulados se habrán de diseñar sistemas totales, favorecer estructuras y organización accesibles (recursos, contexto y en actitudes) y desarrollar los mecanismos de compensación, que hagan posible la educación y participación de las personas con discapacidad en una comunidad universitaria inclusiva.

- Desarrollar Unidades o Servicios de Atención a Estudiantes con Discapacidad, que no sólo palien la falta legislativa y de intervenciones, sino que cubran la atención y apoyo a sus necesidades, servicio considerado y ubicado, como un servicio más que presta la comunidad universitaria a su alumnado.

- No debe cuestionarse a priori, la capacidad o suficiencia de cualquier estudiante con discapacidad, para realizar los estudios que haya elegido. Le asiste el derecho de hacer la carrera que vocacionalmente haya deseado, facilitándose el acceso y el desarrollo en la misma, así como los apoyos y compensaciones que se precisen. La formación debe estar antes que la instrucción e información específica de la carrera en sí, dejando para su término la evaluación de su preparación como profesional. De esa forma se hace valoración y no juicios de valor (previos), que frenan la progresión y el desarrollo de la persona y su carrera.

- Que, en una visión tradicional, los objetivos sean resultados o metas a conseguir y que estén determinados por los contenidos a aprender, no resta consideración a otra visión paralela, de desarrollo y adaptación curriculares, sin que por ello disminuya la preparación académica, ni el nivel de excelencia; por el contrario, quizá se gane en un mayor nivel de formación personal y cultural, además de lo específico profesional. ¿Es necesario argumentar que el alumnado con discapacidad, ha superado cursos, etapas y procesos selectivos en general, con apoyos sí, pero con más dificultades u obstáculos que el resto de estudiantes? Siendo justo pensar que tienen los mismos derechos, también lo es expresar que tienen necesidades especiales a las que atender, y es precisamente esta atención, la que demuestra la justicia (siempre distributiva y no igualitaria) de la Institución que los acoge.

Finalmente, si hablar de discapacidad implica no centrarse en la limitación individual o deficiencia, sino en el desajuste del entorno social hacia la persona y sus necesidades, en la Universidad, esta apreciación conlleva evitar que se dude de la capacidad de las personas con discapacidad para desarrollarse y enfrentarse a la sociedad, ya que la ejecución de cualquier acción, precisa tanto de habilidad y capacidad como disponibilidad y oportunidad. ¿Tienen los alumnos con discapacidad las condiciones oportunas para ese desarrollo? Sólo cuando se conteste afirmativamente a esta cuestión, podremos valorar adecuadamente sus capacidades y referirnos a los alumnos con discapacidad universitarios, en reciprocidad con sus compañeros sin ella. Desde cualquier Servicio de Apoyo al Alumnado con discapacidad, se debe tratar y trabajar para ello, buscando tan sólo algo tan sencillo y sujeto a derecho, como el principio de igualdad de oportunidades, con el que, a su vez, desarrollar una docencia, aprendizaje y vida universitaria en un marco inclusivo y de normalización. 


\section{Referencias bibliográficas}

Agencia Europea para el Desarrollo de la Educación Especial (1998). Integración en Europa: Provisión para alumnos con necesidades educativas especiales. Tendencias en 14 países. Comisión Europea. Dinamarca. URL: http://www.european-agency.org

Resolución del Consejo de 5 de mayo de 2003, sobre la igualdad de oportunidades en educación y formación para los alumnos y estudiantes con discapacidad. Diario Oficial de la Unión Europea. DOC 134/04 (6-7).

Hegarty, S. (1996). "La Educación Especial en Europa". Revista Española de Pedagogía. No 204. 345-360.

Ibáñez, P. (2002). "La UNED ante la discapacidad". A Distancia (monográfico Universidad y Discapacidad). Vol. 20. 1. (20-27).

Ley Orgánica 4/2007, de 12 de abril, por la que se modifica la Ley Orgánica 6/2001, de 21 de diciembre, de Universidades. BOE no 89 de 13/04/07. (89-109).

Luque, D. J.; Rodríguez, G. (2005). Accesibilidad y adaptación curriculares al alumnado con discapacidad en la Universidad. Una reflexión docente. Comunicación presentada en el I Congreso Nacional de Universidad y Discapacidad. Salamanca, 24 y 25 de noviembre de 2005.

Luque, D. J.; Elósegui, E.; Rodríguez, G. (2005). Consideraciones para un proyecto de Unidad de Apoyo a Personas con discapacidad, en la Universidad de Málaga. Comunicación presentada en el I Congreso Nacional de Universidad y Discapacidad. Salamanca, 24 y 25 de noviembre de 2005 .

Luque, D. J. (2006). Orientación Educativa e Intervención Psicopedagógica en el alumnado con discapacidad. Análisis de casos prácticos. Málaga. Aljibe.

Luque, D. J., y Rodríguez, G. (2006). "Aspectos de intervención psicopedagógica en el alumnado universitario con discapacidad". Ponencia I ${ }^{\circ}$ Congreso Internacional de Psicopedagogía. Melilla.

Luque, D. J.; Rodríguez, G.; Romero, J. F. (2005). “Accesibilidad y Universidad. Un estudio descriptivo". Intervención Psicosocial, Vol. 14. 2., 209-222.

MECD (2003). La Integración del Sistema Universitario Español en el Espacio Europeo de Enseñanza Superior (Documento-Marco). Madrid. MECD.

OMS (1983). Clasificación Internacional de la Deficiencia, Discapacidad y Minusvalía. Madrid. IMSERSO.

OMS (2001). Clasificación Internacional del Funcionamiento, la Discapacidad y la Salud. Madrid. Ministerios de Trabajo y Asuntos Sociales.

\section{ANEXO}

Disposición adicional vigésima cuarta. De la inclusión de las personas con discapacidad en las universidades.

«1. Las Universidades garantizarán la igualdad de oportunidades de los estudiantes y demás miembros de la comunidad universitaria con discapacidad, proscribiendo cualquier forma de discriminación y estableciendo medidas de acción positiva tendentes a asegurar su participación plena y efectiva en el ámbito universitario.

2. Los estudiantes y los demás miembros con discapacidad de la comunidad universitaria no podrán ser discriminados por razón de su discapacidad ni directa ni indirectamente en el acceso, el ingreso, la permanencia y el ejercicio de los títulos académicos y de otra clase que tengan reconocidos. 
3. Las universidades promoverán acciones para favorecer que todos los miembros de la comunidad universitaria que presenten necesidades especiales o particulares asociadas a la discapacidad dispongan de los medios, apoyos y recursos que aseguren la igualdad real y efectiva de oportunidades en relación con los demás componentes de la comunidad universitaria.

4. Los edificios, instalaciones y dependencias de las universidades, incluidos también los espacios virtuales, así como los servicios, procedimientos y el suministro de información, deberán ser accesibles para todas las personas, de forma que no se impida a ningún miembro de la comunidad universitaria, por razón de discapacidad, el ejercicio de su derecho a ingresar, desplazarse, permanecer, comunicarse, obtener información u otros de análoga significación en condiciones reales y efectivas de igualdad. Los entornos universitarios deberán ser accesibles de acuerdo con las condiciones y en los plazos establecidos en la Ley 51/2003, de 2 de diciembre, de igualdad de oportunidades, no discriminación y accesibilidad universal de las personas con discapacidad y en sus disposiciones de desarrollo.

5. Todos los planes de estudios propuestos por las universidades deben tener en cuenta que la formación en cualquier actividad profesional debe realizarse desde el respeto y la promoción de los Derechos Humanos y los principios de accesibilidad universal y diseño para todos.

6. Con arreglo a lo establecido en el artículo 30 de la Ley 13/1982, de 7 de abril, de Integración Social de los Minusválidos y en sus normas de desarrollo, los estudiantes con discapacidad, considerándose por tales aquellos comprendidos en el artículo 1.2 de la Ley 51/2003, de 2 de diciembre, de igualdad de oportunidades, no discriminación y accesibilidad universal de las personas con discapacidad tendrán derecho a la exención total de tasas y precios públicos en los estudios conducentes a la obtención de un título universitario.»

LEY ORGÁNICA 4/2007, de 12 de abril, por la que se modifica la Ley Orgánica 6/2001, de 21de diciembre, de Universidades. (BOE $n^{\circ} 89$ de 13/04/2007).

Fecha de recepción: 28-04-06

Fecha de revisión: 21-04-08

Fecha de aceptación: 21-05-08 